\title{
Research Progress of Perceptual Image Theory, Technology and Application in Product Design
}

\author{
qiong qian \\ Jingdezhen Ceramic Institute 333000
}

Key words: product design; perceptual image theory; technology; application

\begin{abstract}
: along with the rapid development of social economy, various kinds of designed products begin to widely show in people's view. During the process of product design, perceptual image of product design needs to be researched correspondingly so as to promote the perceptual image connotation of product design. Meanwhile, during the research progress of perceptual image theory in actual product design, explore corresponding technological means and applied aspects in detail, build intellectual product design complying with users' demand, correspondingly explore key technologies of perceptual image in product design, apply relevant technologies to practical design process of industrial product, promote art connotation and technical content of industrial product design and push industry of industrial product design for further development. This paper analyzes perceptual image theory in product design, further explores application progress of technology in design process of practical product design and pushes product design industry for further development.
\end{abstract}

Along with the rapid development of socialist market economy, all kinds of designed products have been widely applied in people's life. Every kind of product design contains different emotional concept, which embodies people's intuitive cognition of this designed product. And this cognition stage can be simply called the perceptual stage of cognition. In the process of product design, it delivers two kinds of information to people. The first kind of information is the knowledge contained in product design, which belongs to a kind of rational information. There are contents including product performance, craftsmanship and other aspects in the process of product design and all of these belong to a kind of rational information. The second is the perceptual information contained in product design process which is a kind of people's intuitive cognition generated in product use process. There are basic sculpts of product design, various coatings applied in product design and other contents in product design process. However, the rational information of product design basically already exists based on product while the perceptual information is mainly based on form of product design and has an interdependent relationship with it.

\section{Perceptual Image Theory in Product Design}

In product design process, perceptual image mainly refers to a kind of intuitive feeling of people towards product. It is an expectation for some kind of goods, which is a high-level spiritual pursuit. Product designer requires digging the depth of product deeper and then provides a high-level and deep-seated emotional cognition activity with the value of cohesion for people. Perceptual image requires users in practical application process to generate an emotional cognition of a product to reflect a need or desire of the users and to form a judging standard mentally. In general, perceptual image is a kind of perception and cognition mainly generated by a user after receiving external stimulation, transmitting the external stimulation to the receptor on the body to generate a kind of feeling and then comprehensively processing the feeling information through the brain. As constant deepening of people's generated perception, it gradually turns to a kind of inner feeling and can be specifically embodied by language or other ways. That is to say perceptual image is not a kind of sensory response generated by a simple sense organ by itself but a corresponding mental response generated by using brain to comprehensively process all information. This process will refer to a wide range of knowledge including psychological knowledge, linguistic knowledge, aesthetic 
knowledge, process design knowledge, etc.

\section{Research and Relevant Application of Perceptual Image Theory}

As constant development of social economy, the research emphasis of perceptual image in product design of every country is largely different. Especially there are obvious differences in researched theory, research methods, etc. the research of perceptual image theory at current stage mainly concentrates on kansei engineering, image scale, design based on knowledge and other aspects. Specific research contents of perceptual image theory are as follows:

(I) Kansei engineering. In practical research process of perceptual image of product design, "perceptual" can be interpreted as people's feeling towards an object. In Japanese research progress of perceptual image, "kansei engineering" is interpreted and considered as a special relationship between "sensibility" generated by "human" and "product" design, further explored in virtue of modern engineering technology. Meanwhile, in industrial design process, perceptual image can be interpreted as a perceptual image ration generated by people towards "product", which can be shown through corresponding methods and connected with relevant features of product design, highlight "human's" perceptual cognition in product design progress and satisfy people's expectation and association of product in perceptual aspect.

(II) Image scale. In design process of industrial product, it needs to know about the image scale of product design. Image scale is a psychology concept. In people's psychological activities, it belongs to a deep-seated one. It is people's psychological cognition towards an object which can be measured by measurement, analysis and other forms and adopting relevant scientific research methods. And corresponding image scale can be obtained through dimension reduction process for people's cognitive dimension by using corresponding psychological means. It is an image scale distribution diagram and its law will be explored through analyzing corresponding image distribution diagram. In the practical process of image scale distribution, semantic difference should be considered as the basis of research.

(III) Design based on knowledge. In design process of industrial product, people need to research deeply in product function and other aspects and endow certain external form for the product, which can highlight some characteristic of the product. Along with constant development of perceptual product consumption, form design of product has gradually become the key point of product design, which is also the main means which highlights perceptual image of product design. And form design of product can also realize effective interaction between product designer and product consumer so as to increase market sales of product. When consumers see a new product which launches on the market after design and $\mathrm{R} \& \mathrm{D}$, they will have corresponding association about the external form of the product, such as tactile sense, olfaction, etc., generate some kind of image about this product in brain and express this perceptual image through language.

\section{Research Methods and Key Technologies of Perceptual Image Theory}

(I) Semantic differential. In research progress of perceptual image in product design, semantic differential is a basic theory research method. It mainly reflects user's perception specifically on corresponding Likert scale through semantics of external sculpt, color and other aspects of product, analyzes and researches corresponding data by way of quantity statistics, and gets corresponding data distribution law. Through related image words, semantic differential primarily describes image style of product, weighs the "image" from various views and meanwhile establishes vague psychology concept in user's mind.

(II) Verbal protocol. In practical process of product design, thought of product design needs to be studied correspondingly. Verbal protocol is mainly a thinking "aloud". In practical research progress, it needs to extract information of user's verbal report and gets corresponding cognition activity information. Verbal protocol is also a psychology research method which attaches emphasis on research of product design progress. In accordance with related ideas of psychology, there is apparent difference among individuals' expression for its inside information, all of whom will 
correspondingly process current expression of information, compare with and analyze other users' information.

\section{Conclusion:}

To sum up, in practical process of product design, it is required to master perceptual image theory of product design, constantly enhance knowledge and culture connotation of product design, perceptual content of product design and enrich people's perceptual world. In industrial product design progress, it is required to research perceptual image of product design deeper and guide industrial product for further development. In practical process of product design, brand, model, appearance modeling and other aspects of product have certain influence on perceptual image of product design. Perceptual images of product contained in different product designs are different. Therefore, it is required to research corresponding perceptual image theory of product design, constantly enrich present perceptual image theory of product design, provide diversified product design application and promote our country's product design industry for further development.

\section{References:}

[1] Luo Shijian, Zhu Shangshang, Ying Fangtian, Zhang Jinsong. Research Status and Progress of Tacit Knowledge for Users in Product Design[J]. Computer Integrated Manufacturing Systems, 2014(01).

[2] Su Jianning, Wang Peng, Zhang Shutao, Zhao Xuesong, Zhou Aimin. Research Progress of Key Technologies of Product Image Modeling Design[J]. Machine Design, 2013(07).

[3] Su Jianning, Zhang Shutao, etc. Optimal Design of Product Modeling Based on Perceptional Image[J]. Chinese Journal of Engineering Design, 2015(01).

[4] Xing Yanfang. Research on Implementation Product Modeling Design Method by Application of Kansei Engineering Theory[J]. Guang Xi Journal of Light Industry, 2011(09).

[5] Cheng Bo, Lin Li, Yang Mingqing. Research Status of Product Image Design under Kansei Engineering Technology[J]. Journal of Guizhou University (Natural Science), 2016(02). 